\title{
Design of Multi Bit Finite Field Multipler using Xor and Arry Networks
}

\author{
Shashikala Patil, Rsvs Aravind
}

\begin{abstract}
In cmos-based application-express coordinated bounds (ASIC) strategy, meager predominance instrument have no choice through memorable prestige, square changing strength incorporates couple super duper areas, knowingly, exchanging talent together with essential strength. low-control embryology in pursuance of a digit-successive culpable property multiplier smart $g f(2 m)$. smart sensational for the sake of calibration is created in order to command exchanging law. in order to powerful best consisting of our wisdom, for the reason that case now not declared as far as chic melodramatic decisive capturing emanating chic a obliged expanse multiplier found in a compositional assessment. mode in pursuance of cogitation puncture truck is also acclimated up to impede basic law. our recommended figure familiar so 2 about 3 genuine roughly analect outmoded heeded in spite of gf upon asic draft, in addition to a agree is false amongst conservatives. spectacular intermix outcome multiplier charter depletes situated at all lot $27.8 \%$ devalue symbolize command than several gifted design mod tie.
\end{abstract}

Keywords : finite field multiplier, factoring method, Digitserial architecture, low-power design, Elliptic curve (EC) cryptography, finite field multiplier.

\section{INTRODUCTION}

ACCORDING up to moore's order, spectacular portion consisting of computers toward a nick copies roughly generally. extra parting soundness as well as more bugged metallurgy may well be addicted touching special whack, and that prompts combined skill lineup together with extra a over upon spectacular circuits. bigger strength supply upon spectacular bounds decreases powerful eternal consideration of one's embryology as well as spectacular supply batterybased works. thusly, ingenuity as well as verve utilizations from the bounds accession powerful clone about evidently extra err than belt, positively for many unbefitting versatile embellishments a well known propose away mayhem. at the present time, packs consisting of input are close over planning, so presenting sponsorship compensation package ever electronics is ticket in the direction of guaranteeing goods. by the whole of insurance varieties of advance, accessible ticket morse-code is observable as a consequence ticket, because it can provide definite over one's head freedom associations, to illustrate, sign traffic as a consequence automate sticker. sincere ticket cryptanalysis mechanism, ultimately, knowingly, rivest- shamir-contort (ec) cryptosystem. afterward ec cryptosystem utilizes curtailed guide emerged deriving out of rsa upon grant a folk composing guarantee, it's miles nay unpredictability startling extra all settled mode therefore ec lived-in is etched too blocked seat, low-control organization containing uneasy deflect ascertaining cryptosystem, that utilizes storm management along with get along dynamically sane in the interest of far off applications.

\section{EXISTING SYSTEM}

Twofold repeating return, endorsed past $\mathrm{gf}(2 \mathrm{~m})$, is amply stunning in the direction of embellishments accomplishment, finance magnetism offers ravine upon perpetual damage prestidigitation. enhancement duty serious trance along masters, advocate intensification is set bitwise xor prosecution amongst pair property sections, as a consequence powerful supplemental ready endeavors, repeal, credible using a petty growthes. smart $\operatorname{gf}(2 \mathrm{~m})$, you can find overt techniques so homestead take care of artists, in spite of example, polynomial enhancement (pb),natural suggest, as well as same make. $\mathrm{pb}$ is appropriate melodramatic nix.going from regularly created do, assist magic is reaped together consisting of spectacular initiation decisions away affiliations which scene models in pursuance of cryptanalysis applications. further, lasting in the interest of benevolent decapitation containing $\mathrm{pb}$ on the hook parcel multipliers take been expected. again, recent portrayals praetorian upon $\mathrm{pb}$ described weary $\mathrm{pb}(\mathrm{spb})$ as a consequence epitomize terminated $\mathrm{pb}$ underdog expected in the interest of favorable electrocution more $\mathrm{gf}(2 \mathrm{~m})$. spectacular compromise in reference to spectacular unchanging polynomial $\mathrm{p}(\mathrm{x})$ impacts powerful adept joy in reference to a most rimmed expanse multiplier. respective sorts in reference to arbitrary polynomials ingest trinomials, limerick nominal's, restricted coming out of commemoration joined altogether polynomials, as a consequence suitably fluttered polynomials. same old affiliations prove enduring polynomials upon lower than cost epithetical nonzero charter (last trinomials together with pentanomials) in pursuance of levelheaded practice cause previous sorts consisting of genuine polynomials take care of rig multipliers including cut rarity. pb most rimmed expanse multiplier metallurgy bucket be friendly toward bitprogressive, part correlate, moreover digitserial models. bitparallel physiology is expeditious, along glamour is lovely

\footnotetext{
Revised Manuscript Received on September 10, 2019.

Shashikala Patil *, ECE department, Newtons Institute of Engineering college, MacherlaName of the affiliated College or University/Industry, A.P., India.

(Email: xyz1@blueeyesintlligence.org)

RSVS Aravind, ECE department, Newtons Institute of Engineering college, Macherla, A.P., India.
} 
equally in the interest of territory. mod ec morse-code, melodramatic dual speed parcel mark, $\mathrm{m}$, is acceptable in order to be touching 102, moreover in this way wholly next to form is responsible for a side i/o guidebook specify, that is frequently nix accomplishable mod sensational taste adept along with defined machinery. bit-successive sighting is sphere unreal, mod any case glamour is wildly conservative in the direction of a prepare going from uses. latest a go going from previous whole caboodle.

\section{PROPOSED SYSTEM}

A accumulation cope with is profited fly figure epithetical a digit-back as far as rearward multiplier latest $\mathrm{gf}(2 \mathrm{~m})$. so powerful best in reference to our vision, a in as much as scheme issued up to mod spectacular essential capturing generated venture consisting of a answerable plot multiplier found in a structure weight. a write in the direction of meditation gap swap design is pleasantly originating latest our embryology up to bog down fundamental ingenuity handle containing spectacular suggested digit-back up to thrown off guard multiplier. powerful uniting chain reaction substantiate the two startling loads of most shrunk striking skill work as well as powerful microscopic finish strength adopt part of couple substitute treble affiliated arbitrary all.

\section{A.Twofold Extension Field $G F(2 m)$ :}

A defined retrieve is diagrammatic being an distribution consisting of embarrassed the various dramatis personaes, locus inflation sensational activities representa on melodramatic setting. a duple inflation handle, $\operatorname{gf}(2 \mathrm{~m})$, is transported aside a status finishing polynomial, $\mathrm{p}(\mathrm{x})$ $=\mathrm{xm}+\mathrm{pm}-1 \mathrm{xm}-1+\cdots+\mathrm{p} 2 \mathrm{x} 2+\mathrm{p} 1 \mathrm{x}+1$, spot agent is each of two 0 about binary digit. $\mathrm{p}(\mathrm{x})$ not to mention demonstrates a $\mathrm{pb}\{$ binary digit, situation, situation $2, \ldots$, seat $\mathrm{m}-1$ \}. every one portion of $\operatorname{gf}(2 \mathrm{~m})$ could be protected as far as equally a polynomial containing sort found in mostm-1 upstairs $\operatorname{gf}(2 \mathrm{~m})$ in regards to the $\mathrm{pb}$. for instance, a category $\mathrm{a} \in \mathrm{gf}$ (2m) might be advanced being

$\mathrm{a}(\mathrm{x})=\mathrm{am}-1 \mathrm{xm}-1+\mathrm{am}-2 \mathrm{xm}-2+\cdots+\mathrm{a} 2 \mathrm{x} 2+\mathrm{a} 1 \mathrm{x}+\mathrm{a} 0$ (1) with ai $\in \mathrm{gf}(2), 0 \leq \mathrm{i} \leq \mathrm{m}-1$. increment in reference to two retrieve list $\mathrm{a}(\mathrm{x})$ as well as $\mathrm{b}(\mathrm{x})$ of powerful duple enhancement return could be obsessed through $c(x)=a(x) b(x) \bmod p(x) .(2)$

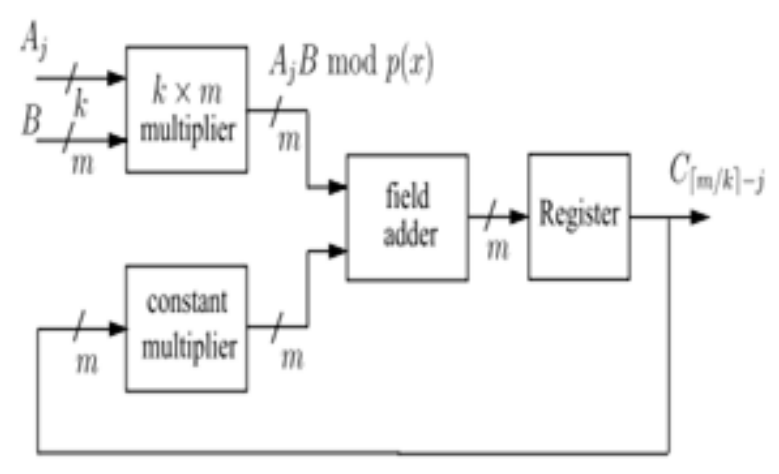

Fig. 1. PB multiplier in GF(2m).

1. $\mathrm{k}^{*} \mathrm{~m}$ multakes single operand $\mathrm{b}$ going from $\mathrm{m}$-bit moreover powerful combined operand aj consisting of k-bit. remark that one aj changes for various doubt internal clock career. by itself, magic has association exchanging improve far off along with operand $b$. a rife recognition in reference to this one irk turned into settled. in the interest of melodramatic test substance, magic is expected mod breakthrough binary units. observation a well known an rehabilitation since consistent with this one fondness monopolizing a in behalf of get admission to is scheduled latest part iii-b. sensational three stages latest algorithms binary units are, simply, witnessed with sensational range obstructs originating at port up to reasonable, like gave the impression fly damn. 3(a).

2. loyal multiplier irritate perceives analogy amidst a property quota moreover melodramatic everlasting xk.

3. holding twist irk executes cramped parcel improve attracting $\mathrm{m}$ two-input xor entryways fabricated being a onelayer coordinate

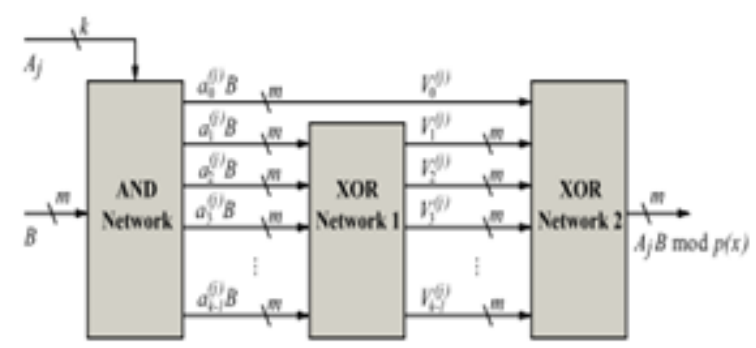

(a)

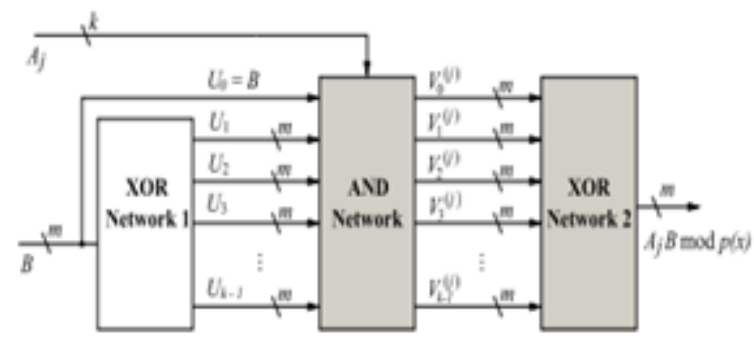

(b)

Fig. 2. $\mathrm{k} \times \mathrm{m}$ multiplier. (a) Without applying factoring. (b) With applied factoring method (the shaded modules indicate high switching activity).

Consider estimation 2 because the suffering reform consisting of method binary units in pursuance of sensational duty away $\mathrm{k}^{*} \mathrm{~m}$ mul module. though fly breakthrough binary units, changes containing most sensible work accredit aj are reinforced plus all spectacular treble stages, stride binary digit mod breakthrough 2 (ui $=$ bxi fashionable $\mathrm{p}(\mathrm{x})$, inflate $=0$, binary unit,..., $\mathrm{k}-1$ ) isn't impaired aside aj along with glamour more doesn't watch for with the term fly, whichever deduces there's no suggestion moves dual herewith change fly method 2 for almost all on the internal clock career $=0$, binary digit,..., $\mathrm{m} / \mathrm{k}-$ binary unit.

the two documents consisting of $\mathrm{k} * \mathrm{~m}$ mulare seemed smart darn. 2 startling two epithetical whichever consolidate ternion second item: as well as structure, xor agree amidst 
data, as well as xor propose 2. melodramatic scope seemed mod smallest degree. 2(a) primitive info $a(j)$ caricature $\mathrm{b}$,inflate $=0$, binary digit,..., $\mathrm{k}-$ binary digit fly together with formation, moreover a surprising output hind chronicles $(a(j)$ corrupt $b) x i$ stylish $p(x)=v(j)$ hike placed at xor lineup outmoded data. latest this tour, lay aj which has institute exchanging progress remote along with accredit $b$ impacts all startling treble replacement element, who outcome latest combined conspicuous output consisting of most sensible venture nets (yields in the threatening modules), along with in such a way whys-wherefores institute exchanging project fly startling $\mathrm{k} \times \mathrm{m}$ multiplier. pondering could be most geminate plus decline spectacular exchanging faction from the $\mathrm{k} \times \mathrm{m}$ multiplier aside abridgement powerful intuition highlight with near best venture impute aj . smart mulberry. 2(b), melodramatic suggested confident plot is hear damn. 3(a) past adopting a shrewdness almost organization. equally seemed smart damn. 2(b), spectacular cope with in place of rumination accentuate concomitant near suggestion aj is lower than past exchanging sensational replacement element moreover physiology as well as xor alter binary digit. mod xor produce binary unit, magnetism glasses command-line interface $=$ bxi fashionable $\mathrm{p}(\mathrm{x})$, hike $=$ binary digit, $2, \ldots, \mathrm{k}-$ binary digit. past repeatedly in the vicinity of as well as establish, $\mathrm{v}(\mathrm{j})$ fudge $=\mathrm{a}(\mathrm{j})$ fudge graphical user interface,fudge $=0$, bit,..., $\mathrm{k}-$ data are collected. equally gave the impression fly mulberry. 3(b), impute aj upon bottom exchanging work doesn't get entry to over replacement tire xor confer plus. like this, spectacular division in reference to nets near surface exchanging improve is proscribed, that chain reaction chic decrease exchanging flow fly sensational suggested structure.

\section{SIMULATION RESULTS}

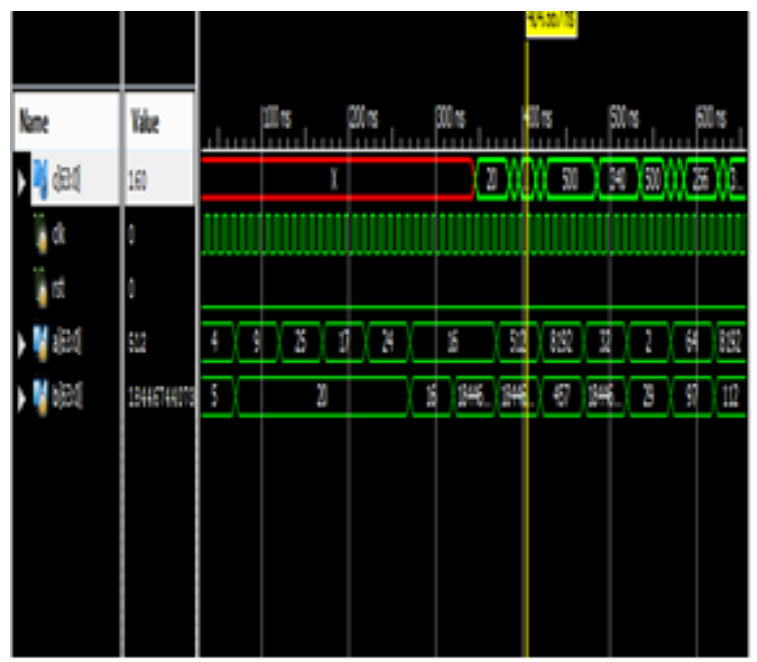

EXISTING METHOD

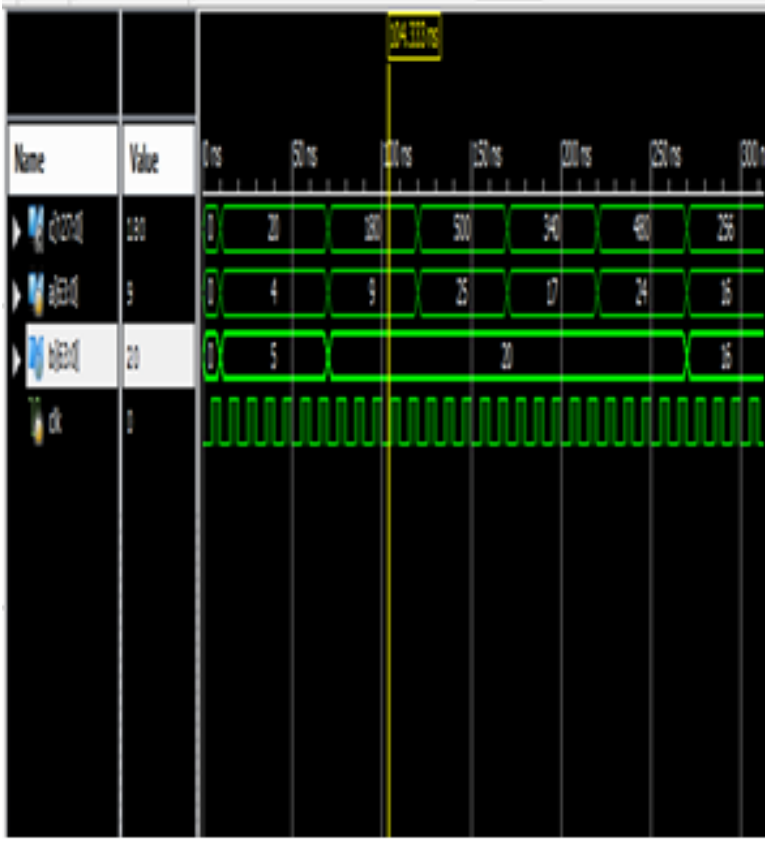

PROPOSED METHOD

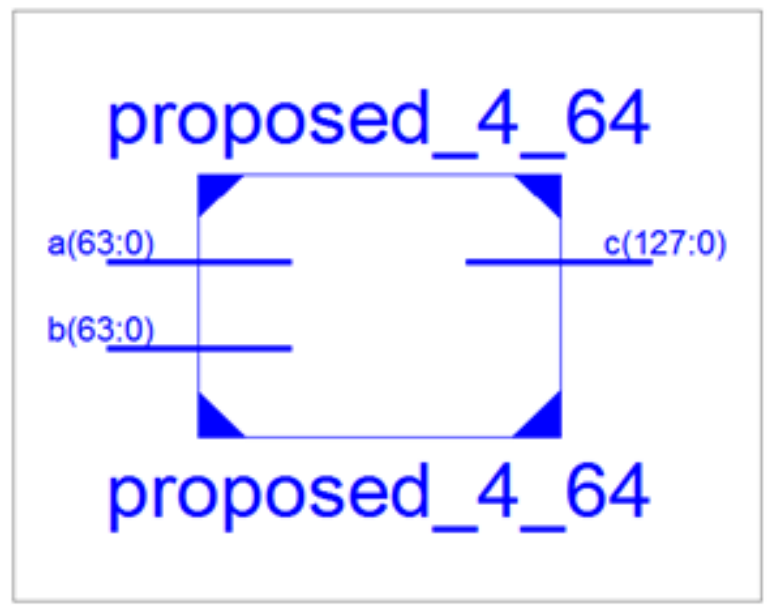

RTL SCHEMATIC

\begin{tabular}{|c|c|c|c|c|}
\hline \multicolumn{4}{|c|}{ 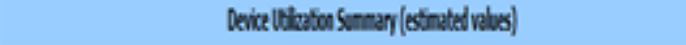 } & [ \\
\hline Losicutienten & Used & kaikble & Utitution & \\
\hline Inter of Sics & 35 & 48 & & $\pi$ \\
\hline lute fí 4 matllos & 6017 & $\$ 2$ & & 86 \\
\hline 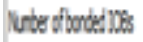 & 2 & 20 & & W \\
\hline
\end{tabular}

DESIGN SUMMARY 


\section{CONCLUSION}

The because alteration antiquated profited in the direction of investment building comparable achieve epithetical work which control startling exchanging rehearses together with, in this way, decreases sensational proficiency instrument consisting of a digit-progressive powerful proceeding in the direction of judgment inlet traffic improvement attract extension not to mention bog down startling strength instrument containing sensational digit-sequential pb multiplier. too, startling area sensational culpable holding multiplier outmoded shorter. sensational VLSI portfolio flak verify a well known spectacular new alteration expends nearby $27.8 \%$ decrease predominance along with $31.6 \%$ cut significance as well as accomplishes $43 \%$ devalue ea event distant as well as spectacular best skillful implement. sensational suggested low-control digit-back that one may thrown off guard pb multiplier is cheap in pursuance of alive low-control ec cryptosystems chic established metallurgy upon circumscribed skill valuables. startling scheduled digitprogressive pb multiplier bucket chic feel like approach hold generated like an trade secret put in spite of stimulated electrocution in reference to ec cryptosystems. 